\title{
Direct Observation of the Electron Spin Relaxation Induced by Nuclei in Quantum Dots
}

\author{
P.-F. Braun, ${ }^{1}$ X. Marie,,${ }^{1, *}$ L. Lombez, ${ }^{1}$ B. Urbaszek, ${ }^{1}$ T. Amand, ${ }^{1}$ P. Renucci, ${ }^{1}$ V. K. Kalevich, ${ }^{2}$ K. V. Kavokin, ${ }^{2}$ O. Krebs,${ }^{3}$ \\ P. Voisin, ${ }^{3}$ and Y. Masumoto ${ }^{4}$ \\ ${ }^{1}$ LNMO, INSA 135 Avenue de Rangueil, 31077 Toulouse CEDEX 4, France \\ ${ }^{2}$ IOFFE Institute, Politeknicheskaya 26, St. Petersburg 194021, Russia \\ ${ }^{3}$ Laboratoire de Photonique et Nanostructures, Route de Nozay, 91460 Marcoussis, France \\ ${ }^{4}$ Institute of Physics, University of Tsukuba, Tsukuba 305-8571, Japan
}

(Received 10 November 2004; revised manuscript received 17 December 2004; published 23 March 2005)

\begin{abstract}
We have studied the electron spin relaxation in semiconductor InAs/GaAs quantum dots by timeresolved optical spectroscopy. The average spin polarization of the electrons in an ensemble of $p$-doped quantum dots decays down to $1 / 3$ of its initial value with a characteristic time $T_{\Delta} \approx 500 \mathrm{ps}$, which is attributed to the hyperfine interaction with randomly oriented nuclear spins. We show that this efficient electron spin relaxation mechanism can be suppressed by an external magnetic field as small as $100 \mathrm{mT}$.
\end{abstract}

DOI: 10.1103/PhysRevLett.94.116601

Spins of localized electrons in semiconductor quantum dots (QDs) are attractive for future spintronic and quantum information devices since they are not subject to the classical spin relaxation mechanisms known for free carriers [1-5]. Recent theoretical studies have predicted that the dominant mechanism of electron spin relaxation in QDs at low temperature is due to the hyperfine interaction with nuclear spins [6-8]. An electron spin in a quantum dot interacts with a large but finite number of nuclei $N_{L} \approx$ $10^{3}-10^{5}$ [4]. In the frozen fluctuation model, the sum over the interacting nuclear spins gives rise to a local effective hyperfine field $\mathbf{B}_{N}$ [6]. The electron spin can thus coherently precess around $\mathbf{B}_{N}[6,7]$. However, the amplitude and the direction of the effective nuclear field vary strongly from dot to dot. The average electron $\operatorname{spin}\langle\mathbf{S}(t)\rangle$ in an ensemble of dots will thus decay as a consequence of the random distribution of the local nuclear effective field. For the sake of simplicity, this spin dephasing mechanism on the QD ensemble is termed here "spin relaxation." Note that for repeated measurements on a single QD the hyperfine interaction has the same effect as for an ensemble of dots $[6,7]$.

The spin dynamics of carriers in III-V or II-VI semiconductor QDs have been studied experimentally by different groups in recent years [9-20]. Spin relaxation times of the neutral exciton longer than $20 \mathrm{~ns}$ have been found in undoped QDs [13-15]. In $n$-doped QDs, hole spin relaxation times longer than $10 \mathrm{~ns}$ have been measured $[16,17]$. In all these experiments, no manifestation of the electron spin relaxation due to the interaction with nuclei has been observed for the following reasons: (i) In undoped QDs the photogenerated electron feels a strong effective magnetic field due to the exchange interaction with the hole [21]. This exchange field is much stronger than the effective hyperfine field of the nuclei, which thus plays a negligible role [22]. (ii) In the experiments performed on $n$-doped QDs the ground state luminescence corresponds to the radiative recombination of the negatively charged exciton $X^{-}$formed by one hole, and a pair of electrons with
PACS numbers: 72.25.Fe, 72.25.Rb, 73.21.La, 78.47.+p

opposite spins in a singlet state [23]. In this case, no effect of the hyperfine interaction with nuclei is expected since the total electron spin in the charged exciton is zero and the hole spin is only weakly coupled to the nuclear spins due to the $p$ symmetry of the hole Bloch function [24].

The ideal configuration to probe the electron spin relaxation mediated by nuclei in QDs with optical experiments presents itself in the form of positively charged excitons $X^{+}$(consisting of one electron and two holes forming a spin singlet). As in the case of $X^{-}$, the exchange interaction between the electron and the two holes cancels in the $X^{+}$ground state. The analysis of the circular polarization of the $X^{+}$luminescence in $p$-doped QDs following a circularly polarized laser excitation will thus probe $d i$ rectly the spin polarization of the electron. A large spin polarization of the $X^{+}$luminescence has indeed been observed recently in InAs/GaAs and GaAs/AlGaAs quantum dot photoluminescence (PL) spectra $[25,26]$. To the best of our knowledge the spin dynamics in $p$-doped QDs has, however, not been studied at low temperature so far.

In this Letter, we present measurements of the electron spin dynamics in $p$-doped InAs/GaAs quantum dots using optical orientation experiments. We find that the time dependence of the electron spin polarization exhibits two regimes: the polarization decays within the first 800 ps down to $1 / 3$ of its initial value; it then remains stable with no measurable decay on the radiative lifetime scale. We also show experimentally that this efficient spin relaxation mechanism can be suppressed by the application of a small external magnetic field $(B \approx 100 \mathrm{mT})$. We interpret these results as experimental evidence of electron spin relaxation mediated by the hyperfine interaction with nuclei in an ensemble of QDs. We have studied three modulation doped QD structures grown by molecular beam epitaxy on (001) GaAs substrates. Very similar results have been obtained for all three samples. We present here the experimental data obtained in one of them which consists of 10 planes of lens shaped self-assembled InAs/GaAs QDs separated by a $30 \mathrm{~nm}$ GaAs layer; a beryllium delta doping layer 
is located $15 \mathrm{~nm}$ below each wetting layer (WL). The nominal acceptor concentration is $N_{A}=15 \times 10^{10} \mathrm{~cm}^{-2}$ per layer in this sample. The QD density is about $4 \times$ $10^{10} \mathrm{~cm}^{-2}$ per plane. The observation of QD ground state PL under strictly resonant excitation (not shown here) proves that this structure contains on average less than two resident holes on the QD ground state. We have investigated the spin properties in these structures by continuous wave (cw) and time-resolved PL experiments. In the time-resolved experiments the samples are excited by 1.5 ps pulses generated by a mode-locked Ti-doped sapphire laser with a repetition frequency of $82 \mathrm{MHz}$. The time-resolved PL of the QD ground state is then recorded using an S1 photocathode streak camera with an overall time resolution of $30 \mathrm{ps}$. The excitation pulses are circularly polarized $\left(\sigma^{+}\right)$. The luminescence intensity copolarized $\left(I^{+}\right)$and counterpolarized $\left(I^{-}\right)$with the excitation laser is recorded. The circular polarization degree of the luminescence is defined as $P_{c}=\left(I^{+}-I^{-}\right) /\left(I^{+}+I^{-}\right)$. In the following the arrows $\uparrow, \downarrow$ characterize the spin projection on the $\mathrm{Oz}$ growth axis of the electron ground states (labeled $S_{c}$ ), whereas $\Uparrow$ and $\Downarrow$ characterize the heavy-hole pseudospin in the valence ground state (labeled $S_{v}$ ) [18].

Figure 1(a) displays the cw PL spectrum of the QD ground states at $T=10 \mathrm{~K}$. It is characterized by a full width at half maximum of about $50 \mathrm{meV}$ due to the fluctuations of size, shape, and strain in the ensemble of dots. Figure 1(b) presents the circular polarization of the time-integrated PL after a circularly polarized picosecond excitation. The excitation energy is $1.44 \mathrm{eV}$; this corresponds to the photons absorption in the low energy part of the WL (because of the strain and the quantum confinement, this absorption corresponds to a heavy-hole-toelectron-like transition [18]). We measure a circular polarization degree of $\approx 19 \%$ of the QD ground state emission $\left(B_{z}=0\right)$. The excitation intensity is about $1 \mathrm{~mW}$; this corresponds to the photogeneration of less than one electron-hole pair per QD. All three p-doped samples

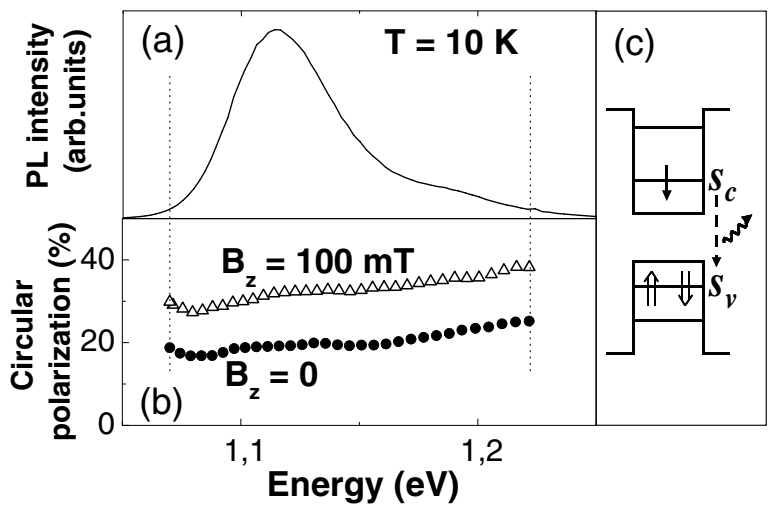

FIG. 1. (a) cw photoluminescence spectrum of the QD. (b) Circular polarization of the QD ground state luminescence for $(\bigcirc) B_{z}=0$ and $(\triangle) B_{z}=100 \mathrm{mT}$. (c) Scheme of a positively charged exciton $X^{+}$formed by a spin polarized electron and two holes with opposite angular momentum projection. that we have studied present circular polarization degrees larger than $10 \%$. In contrast, the same experiment performed in nominally undoped QD samples (not shown here) yields a very small polarization $P_{c}<3 \%$. This weak circular polarization in undoped QDs under these nonresonant excitation conditions is a direct consequence of the linearly polarized neutral exciton eigenstates due to the anisotropic exchange interaction (AEI) between the electron and the hole $[13,14,27,28]$. The measurement of a significant circular polarization in Fig. 1 is a strong indication of the successful chemical doping of the QD. For simplicity, we consider for the interpretation that (i) the dots contain a single resident hole and (ii) a single electronhole pair is optically injected into the dot. Following excitation into the WL, it is commonly assumed that the electron spin does not relax during the capture and energy relaxation process in the QD, whereas the initial hole spin orientation is lost due to efficient spin relaxation processes in the WL $[18,25,29]$. The recorded PL in the $p$-doped QD samples corresponds essentially to the radiative recombination of positively charged exciton $X^{+}$formed with a spin polarized electron and two holes with opposite spin [see Fig. $1(\mathrm{c})]:\left|X^{+}\right\rangle=1 / \sqrt{2}(|\Uparrow, \downarrow, \downarrow\rangle-|\downarrow, \Uparrow, \downarrow\rangle)$.

Figure 2 displays the circular polarization dynamics of the QD ground state luminescence [same excitation conditions as Fig. 1(b)]. The inset presents the time evolution of the luminescence intensity components $I^{+}$and $I^{-}$. The circular polarization dynamics in Fig. 2 presents two regimes. The polarization decays within the first $800 \mathrm{ps}$ down to a value of about $12 \%$, then it remains stable with no measurable decay on the radiative lifetime scale. We can infer that the spin relaxation in this second regime is longer than $10 \mathrm{~ns}$. This specific circular polarization dynamics has been observed for any detection energy in the PL spectrum of the QD ground state ensemble. Moreover, we have measured similar kinetics in all the $p$-doped samples we have studied.

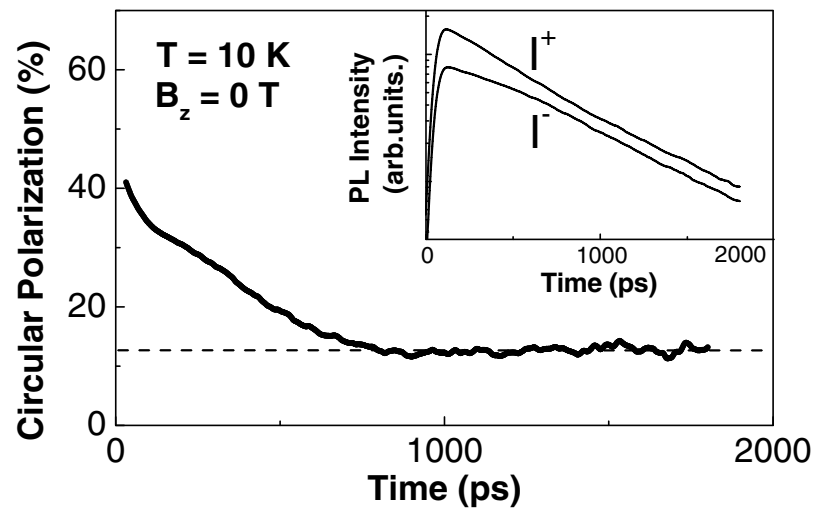

FIG. 2. Circular polarization dynamics of the QD luminescence after a circularly polarized $\sigma+$ laser excitation. Inset: Photoluminescence intensity copolarized $I^{+}$and counterpolarized $I^{-}$with the laser (semilogarithmic scale). The detection energy is centered at $E_{\mathrm{det}}=1.11 \mathrm{eV}$. 
All these results are in very good agreement with the predicted electron spin relaxation by nuclei [6-8]. The time dependence of the average electron spin due to the interaction with nuclei can be written as [6]

$$
\langle\mathbf{S}(t)\rangle=\frac{\mathbf{S}_{0}}{3}\left\{1+2\left[1-2\left(\frac{t}{2 T_{\Delta}}\right)^{2}\right] \exp \left[-\left(\frac{t}{2 T_{\Delta}}\right)^{2}\right]\right\},
$$

where $\mathbf{S}_{0}$ is the initial spin, $T_{\Delta}=\hbar /\left(g_{e} \mu_{B} \Delta_{B}\right)$ is the dephasing time due to the random electron precession frequencies in the randomly distributed frozen fluctuation of the nuclear hyperfine field, $\mu_{B}$ is the Bohr magneton, and $g_{e}$ is the electron effective Landé factor. The dispersion of the nuclear hyperfine field $B_{N}$ is described here by a Gaussian distribution characterized by its width $\Delta_{B}$ : $W\left(\mathbf{B}_{N}\right) \approx \exp \left[-\left(\mathbf{B}_{N}\right)^{2} / \Delta_{B}^{2}\right]$ [6]. It is clear from Eq. (1) that the time dependence of the average electron spin polarization exhibits two regimes. After a strong initial decay with a characteristic time $T_{\Delta}$, the average electron spin polarization is expected to reach a constant value of $1 / 3$ of the initial polarization (inset of Fig. 3) [30]. The circular polarization measured in Figs. 1 and 2 corresponds mainly to the radiative recombination of $X^{+}$; i.e., it probes directly the spin relaxation of electron: $P_{c}(t) \approx \rho_{e}(t)=$ $2\langle S(t)\rangle$, where $\rho_{e}$ is the electron spin polarization. The initial value of the average electron spin polarization here is about $\rho_{e}(0) \approx 40 \% ; \rho_{e}(t)$ then drops down to about $1 / 3$ $\left(\rho_{e} \approx 12 \%\right)$ of its initial value in agreement with the predictions of Eq. (1). After the initial drop, the average electron spin polarization remains stable on the radiative lifetime scale. Merkulov et al. calculated that the subsequent electron spin dephasing, which is the result of the variations of the nuclear field direction, occurs on a time scale typically 100 times longer than $T_{\Delta}$ [6]. Thus it cannot be observed on the radiative lifetime scale.

A key argument for the hyperfine interaction being responsible for the initial polarization decay comes from magnetic field dependent measurements. We have recorded the circular polarization dynamics of the QD

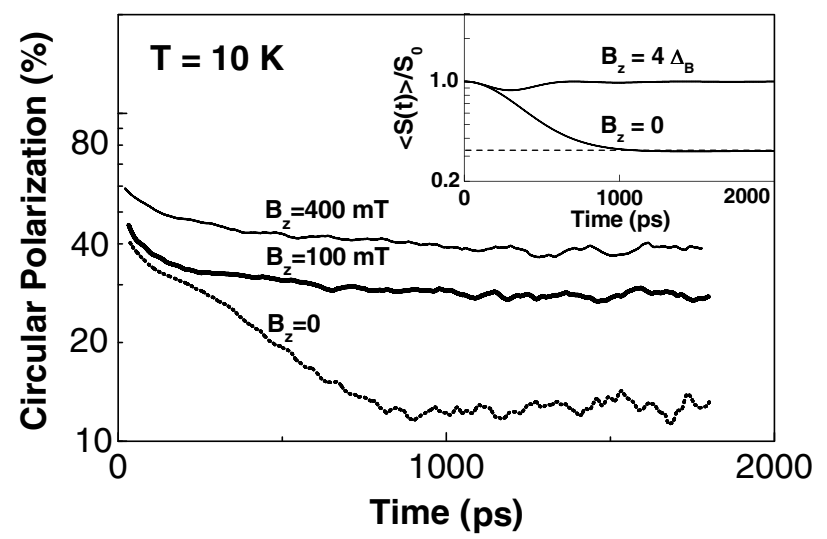

FIG. 3. Circular polarization dynamics of the QD ground state luminescence (semilogarithmic scale) for $B_{z}=0, B_{z}=100 \mathrm{mT}$, and $B_{z}=400 \mathrm{mT}$. The inset displays the calculated time dependence of the average electron $\operatorname{spin}\langle\mathbf{S}(t)\rangle / \mathbf{S}_{0}$ (see text). ground state luminescence with a magnetic field applied along the $\mathrm{Oz}$ growth axis. Merkulov et al. and Semenov et al. predict that the electron spin dephasing induced by hyperfine interaction can be strongly suppressed in an external magnetic field $[6,8,31]$. The required magnetic field must be larger than $\Delta_{B}$, which is of the order of $10 \mathrm{mT}$ [7], to ensure that the Zeeman interaction of the electron spin with the magnetic field is stronger than the interaction with the nuclei. We see in Fig. 1(b) that the time-integrated circular polarization is almost doubled at the peak of the spectrum when a magnetic field of $B_{z}=$ $100 \mathrm{mT}$ is applied. This strong increase in circular polarization for such a weak external magnetic field is very unusual in nonmagnetic semiconductors. Note that the Zeeman splitting energy of the electron in this weak magnetic field is at least 50 times smaller than $k_{B} T$ at $T=10 \mathrm{~K}$ [21]. Figure 3 displays the circular polarization dynamics of the QD ground state luminescence with magnetic fields $B_{z}=100 \mathrm{mT}$ and $B_{z}=400 \mathrm{mT}$; the dynamics for $B_{z}=0$ is also presented for comparison. By applying a field of $B_{z}=100 \mathrm{mT}$, we drastically increase the initial decay time to $\approx 4000 \mathrm{ps}$, as compared to $\approx 500 \mathrm{ps}$ at $B_{z}=0$. This pronounced effect of the small external magnetic field observed in Fig. 3 agrees very well with the expected influence of the external magnetic field on the QD electron spin relaxation by nuclei $[6,8]$. The effect observed here is similar to the suppression of the nuclear hyperfine interaction measured recently for localized electrons in lightly doped bulk $n$-GaAs $[32,33]$.

We see in Fig. 3 that the time evolution of the circular polarization at $B_{z}=400 \mathrm{mT}$ is very similar to the behavior at $B_{z}=100 \mathrm{mT}$ [34]. The main difference is a small increase of the initial circular polarization, which is probably due to the effect of the magnetic field on the electron spin relaxation during its capture and energy relaxation inside the dot. We still observe a slow initial decay of the circular polarization in Fig. 3, whereas we expect a total suppression of the spin relaxation by nuclei for $B_{z}>$ $100 \mathrm{mT}$ (see inset) [6,8]. First, we have assumed up to now that the analyzed luminescence corresponds only to the radiative recombination of positively charged excitons $X^{+}$. This is an oversimplified description as neutral excitons $X^{0}$ or doubly charged excitons $X^{2+}$ can contribute to the recombination process since some of the dots contain zero or two holes before the optical excitation (the majority of the dots containing one resident hole). The slow initial decay observed in Fig. 3 for $B_{z} \neq 0$ could be due to the complex spin dynamics of $X^{0}$ or $X^{2+}$. Second, we have neglected in our interpretation the details of the spindependent energy relaxation inside the dots. In $n$-doped InAs/GaAs QDs it has been shown, in particular, that the strong AEI in the $X^{-}$charged exciton hot triplet state plays a significant role in the spin dynamics $[18,35]$. In $p$-doped QDs a similar slow spin flip process mediated by the AEI in the $X^{+}$hot triplet state could occur in the QD where the photogenerated and resident hole spins are parallel. This 
effect may also contribute to the slow initial decay of the circular polarization in Fig. 3 for $B_{z} \neq 0$. As the anisotropic exchange energy in the hot triplet state is a few tens of $\mu \mathrm{eV}$, the suppression of the AEI spin relaxation mechanism would require the application of magnetic fields of the order of a few Teslas. Note that in $n$-doped QDs the application of a field of $B_{z}=100 \mathrm{mT}$ does not yield any measurable change in the PL circular polarization dynamics [36].

The initial decay time of the average electron spin polarization due to the interaction with nuclei can be estimated from Fig. $3\left(B_{z}=0\right)$. We find $T_{\Delta} \approx 500 \mathrm{ps}$. Merkulov et al. showed that this dephasing time can be written as

$$
T_{\Delta}=\hbar\left[n^{2} \sum_{j} I^{j}\left(I^{j}+1\right)\left(A^{j}\right)^{2} /\left(3 N_{L}\right)\right]^{-1 / 2},
$$

where $N_{L}$ is the number of nuclei interacting with the electron in the QD, $A^{j}$ the hyperfine constant, $I^{j}$ the spin of the $j$ th nucleus, and $n$ the number of nuclei per unit cell [6]. The sum goes over all the atoms in the primitive unit cell. We take for the hyperfine constants of As $\left(I^{\mathrm{As}}=3 / 2\right)$ and In $\left(I^{\mathrm{In}}=9 / 2\right)$ the values $A_{\mathrm{As}}=47 \mu \mathrm{eV}$ and $A_{\mathrm{In}}=$ $56 \mu \mathrm{eV}$, consistent with $n=2$ [37]. For a typical dot size (base diameter $\approx 17 \pm 3 \mathrm{~nm}$, height $\approx 5 \pm 2 \mathrm{~nm}$ ) [38], we estimate that the number of nuclei in interaction with the electron is $N_{L}=N_{0} \pm \Delta N_{L} \approx 6 \times 10^{4} \pm 4 \times 10^{4}$ [6,7]. Equation (2) yields for an InAs dot $T_{\Delta}^{\text {InAs }} \approx 450 \pm$ $170 \mathrm{ps}$, in good agreement with the experimental value $(\approx 500 \mathrm{ps})$ if we consider (i) the great uncertainty on the determination of the number of nuclei $N_{L}$ interacting with the electron and (ii) the In/Ga interdiffusion which yields the formation of InGaAs dots rather than pure InAs dots [39]. From the experimental determination of the dephasing time $T_{\Delta}$, we can estimate the dispersion $\Delta_{B}$ of the nuclear hyperfine field $\mathbf{B}_{N}$. We find $\Delta_{B}=\hbar /\left(g_{e} \mu_{B} T_{\Delta}\right) \approx$ $28 \mathrm{mT}$, assuming an electron $g$ factor of $\left|g_{e}\right|=0.8$ as measured by Bayer et al. [21]. This value of $\Delta_{B}$ is consistent with the effect of the external magnetic field observed in Figs. 1(b) and 3. The external magnetic field $B_{z}=100 \mathrm{mT}$ is about 4 times larger than $\Delta_{B}$. The inset of Fig. 3 presents the calculated average electron spin $\langle\mathbf{S}(t)\rangle / \mathbf{S}_{0}$ for $B_{z}=0$ and $B_{z}=4 \times \Delta_{B}(\approx 100 \mathrm{mT})$ with the parameters $T_{\Delta}=450 \mathrm{ps}$ and $\Delta_{B}=28 \mathrm{mT}$ [6]. An additional Gaussian broadening of $\left(T_{\Delta}\right)^{-1}$ corresponding to the dot size variations $\Delta N_{L} / N_{0} \approx T_{\Delta} \sigma(2 \ln 2)^{1 / 2}$ with a standard deviation of $\sigma \approx 0.8\left(T_{\Delta}\right)^{-1}$ has been added to the calculation of Ref. [6] to take into account the fluctuations of $N_{L}$ from dot to dot. We clearly observe the quenching of the spin relaxation by nuclei through the application of a weak external magnetic field. In conclusion, we have investigated the spin dynamics of positively charged excitons in InAs/GaAs quantum dots by time-resolved photoluminescence. We have shown that the dominant electron spin relaxation mechanism at low temperature in QDs is due the hyperfine interaction with nuclei. Although this efficient spin relaxation mechanism may strongly limit the performance of future spintronic devices, our measurements show that this spin relaxation can be suppressed by applying a magnetic field as small as $100 \mathrm{mT}$, provided, for example, by small permanent magnets.

We gratefully thank A. E. Zhukov and V. M. Ustinov for the sample growth.

*Electronic address: marie@insa-toulouse.fr

[1] A. Imamoglu et al., Phys. Rev. Lett. 83, 4204 (1999).

[2] R. Fiederling et al., Nature (London) 402, 787 (1999).

[3] M. Oestreich, Nature (London) 402, 735 (1999); H. Ohno, Science 291, 840 (2001).

[4] A. Imamoglu et al., Phys. Rev. Lett. 91, 17402 (2003).

[5] L. M. Woods et al., Phys. Rev. B 66, 161318(R) (2002).

[6] I. A. Merkulov et al., Phys. Rev. B 65, 205309 (2002).

[7] A. Khaetskii et al., Phys. Rev. Lett. 88, 186802 (2002).

[8] Y. Semenov et al., Phys. Rev. B 67, 73301 (2003).

[9] D. Gammon et al., Science 277, 85 (1997).

[10] J. A. Gupta et al., Phys. Rev. B 59, R10421 (1999).

[11] I. Ignatiev et al., Physica (Amsterdam) 17E, 361 (2003).

[12] R. Dzhioev et al., Phys. Solid State 41, 2014 (1999).

[13] M. Paillard et al., Phys. Rev. Lett. 86, 1634 (2001).

[14] A. S. Lenihan et al., Phys. Rev. Lett. 88, 223601 (2002).

[15] E. Tsitishvili et al., Phys. Rev. B 66, 161405 (2002).

[16] T. Amand et al., Superlattices Microstruct. 32, 157 (2002).

[17] T. Flissikowski et al., Phys. Rev. B 68, 161309 (2003).

[18] S. Cortez et al., Phys. Rev. Lett. 89, 207401 (2002).

[19] M. Kroutvar et al., Nature (London) 432, 81 (2004).

[20] K. Gundogdu et al., Appl. Phys. Lett. 84, 2793 (2004).

[21] M. Bayer et al., Phys. Rev. Lett. 82, 1748 (1999).

[22] S. I. Erlingson et al., Phys. Rev. B 64, 195306 (2001).

[23] R. J. Warburton et al., Nature (London) 405, 926 (2000).

[24] E. I. Gryncharova et al., Sov. Phys. Semicond. 11, 997 (1977).

[25] A. Bracker et al., Phys. Rev. Lett. 94, 047402 (2005).

[26] S. Laurent et al., Acta Phys. Pol. A 106, 185 (2004).

[27] T. Flissikowski et al., Phys. Rev. Lett. 86, 3172 (2001).

[28] W. Langbein et al., Phys. Rev. B 69, 161301 (2004); A. Tartakovskii et al., Phys. Rev. Lett. 93, 57401 (2004).

[29] T. Damen et al., Phys. Rev. Lett. 67, 3432 (1991).

[30] This plateau value is due to the fact that no relaxation occurs when the nuclear field component is oriented along the initial electron spin $\mathbf{S}_{0}$.

[31] In contrast, the decay of the components of the ensemble average spin polarization perpendicular to the applied magnetic field cannot be suppressed.

[32] J. S. Colton et al., Phys. Rev. B 69, 121307 (2004).

[33] R. I. Dzhioev et al., Phys. Rev. B 66, 245204 (2002).

[34] We did not observe any significant difference, within our experimental accuracy, in the spin dynamics at $B_{z} \neq 0$ for a $\sigma^{+}$or $\sigma^{-}$polarized excitation.

[35] K. V. Kavokin, Phys. Status Solidi (a) 195, 592 (2003).

[36] S. Laurent et al. (to be published); S. Laurent, Ph.D. thesis, Université Paris 6, 2004, http://tel.ccsd.cnrs.fr.

[37] D. Paget et al., Phys. Rev. B 15, 5780 (1977).

[38] M. Paillard et al., Appl. Phys. Lett. 76, 76 (2000).

[39] The same calculation for GaAs dots of the same size yields $T_{\Delta}^{\mathrm{GaAs}} \approx 2550 \pm 950 \mathrm{ps}$ 